\title{
An $85-120 \mathrm{GHz}$ high-gain and wide-band InP MMIC amplifier
}

\author{
Wang Zhi-Ming ${ }^{1 \text { a) }}$, Zhao Zhuo-Bin ${ }^{1}$, Liu Jun ${ }^{1}$, Hu Zhi-Fu' ${ }^{2}$, \\ Sun Xi-Guo ${ }^{2}$, Cui Yu-Xing ${ }^{2}$, Fu Xing-Chang ${ }^{2}$, and Lv Xin ${ }^{1}$ \\ ${ }^{1}$ Beijing Key Laboratory of Millimeter Wave and Terahertz Technology, \\ Beijing Institute of Technology, Beijing 100081, China \\ ${ }^{2}$ Hebei Semiconductor Research Institute, Shijiazhuang 050051, China \\ a)wangzhiming872@163.com
}

\begin{abstract}
This paper presents the development of an $85-120 \mathrm{GHz}$ highgain and wide-band monolithic millimeter-wave integrated circuit (MMIC) amplifier using our own improved 70-nm InP pseudomorphic high electron mobility transistor (PHEMT) with $f_{\mathrm{t}}=247 \mathrm{GHz}$ and $f_{\max }=392 \mathrm{GHz}$. Edgecoupled line is used for DC blocking and radial subs are employed for RF bypass. Shunt RC networks and radial stubs are included in the bias circuitry to maintain amplifier stability. This amplifier is measured on-wafer with a small-signal peak gain of $14.4 \mathrm{~dB}$ at $92 \mathrm{GHz}$ and greater than $11.5 \mathrm{~dB}$ from 85 to $120 \mathrm{GHz}$. The $3 \mathrm{~dB}$ bandwidth is above $35 \mathrm{GHz}$ with a chip size of $1.6 \times 1.1 \mathrm{~mm}^{2}$. To our knowledge, this MMIC amplifier has characteristics of much higher-gain per stage, wider-band and smaller chip size than others at the similar frequency band. The excellent results indicate that this MMIC amplifier has a great potential for pre-amplifier or interstage driving amplifiers applications at W-band or D-band.
\end{abstract}

Keywords: InP, PHEMT, MMIC, amplifier, W-band, D-band

Classification: Microwave and millimeter wave devices, circuits, and systems

\section{References}

[1] P. D. Chow, K. Tan, D. Streit, D. Garske, P. Liu and R. Lai: 1992 IEEE MTT-S International Microwave Symposium Digest (1992) 807. DOI:10.1109/ MWSYM.1992.188110

[2] A. Tessmann: IEEE J. Solid-State Circuits 40 (2005) 2070. DOI:10.1109/JSSC. 2005.854591

[3] W. Deal, X. B. Mei, K. M. K. H. Leong, V. Radisic, S. Sarkozy and R. Lai: IEEE Trans. THz Sci. Technol. 1 (2011) 25. DOI:10.1109/TTHZ.2011. 2159539

[4] HRL Laboratories, LLC: LNA4_110 datasheet (2011) http://mmics.hrl.com/ InP.html.

[5] HRL Laboratories, LLC: PA3_110 datasheet (2011) http://mmics.hrl.com/InP. html. 
Frequency Integrated Circuits (RFIC) Symposium (1999) 99. DOI:10.1109/ RFIC.1999.805248

[7] W. Huei, T. N. Ton, K. L. Tan, D. C. Garske, G. S. Dow, J. Berenz, M. W. Pospieszalski and P. S. Shing: IEEE J. Solid-State Circuits 28 (1993) 988. DOI: $10.1109 / 4.237511$

[8] D. Smith, G. Dambrine and J. C. Orlhac: 2008 Proceedings of the 3rd European Microwave Integrated Circuits Conference (2008) 214. DOI:10.1109/EMICC. 2008.4772267

[9] J. W. Archer and R. Lai: 2000 Asia-Pacific Microwave Conference (2000) 173. DOI:10.1109/APMC.2000.925753

[10] Y. Q. Liu, Z. Zeng, R. Q. Liu, L. H. Han, P. Luan and S. J. Cai: Design and Development of IC China 34 (2009) 579. DOI:10.3969/j.issn.1003-353x.2009. 06.017

[11] L. A. Samoska: IEEE Trans. THz Sci. Technol. 1 (2011) 9. DOI:10.1109/ TTHZ.2011.2159558

[12] Z. M. Wang, X. Lv, Z. F. Hu, X. B. Luo, Y. X. Cui, X. G. Sun, J. H. Mo and X. C. Fu: IEICE Electron. Express 3 (2015) 20141174. DOI:10.1587/elex.12. 20141174

[13] OMMIC: CGY2190UH datasheet (2012) http://www.ommicchina.cn/product/ $\mathrm{html} /$ ?88.html.

\section{Introduction}

W-band (75-110 GHz) and D-band (110-170 GHz) millimeter waves systems have been of great interest for imaging, radar sensor, satellite cross-link and deep space communication applications where they can offer higher resolution, smaller size, high gain, and wider bandwidth [1]. The MMIC amplifiers play a vital role in those systems, in the passive receiving system, the pre-amplifiers can improve the sensitivity, dynamic range and the operating distance of systems. As well as in the transmitting systems, when the source power is limited, the interstage driving amplifiers can provide additional input power to the final stage power amplifier in order to achieve its maximum output power. High-gain, wide-band and low power consumption are necessary for the MMIC amplifiers. Many W-band and D-band even much higher frequency bands MMIC amplifiers have been reported over the world $[1,2,3,4,5,6,7,8,9,10,11]$. Compared with GaAs and other materials based devices, InP-based PHEMTs have shown the excellent performance of highgain, wide-band, and low noise beyond $100 \mathrm{GHz}[3,11]$.

This paper reported the design, fabrication and on-wafer measurement of a twostage $85-120 \mathrm{GHz}$ MMIC amplifier using our previously designed and improved 70-nm InP-based PHEMTs technology. And this technology was also introduced in part 2. This amplifier demonstrated a small-signal peak gain of $14.4 \mathrm{~dB}$ at $92 \mathrm{GHz}$ and greater than $11.5 \mathrm{~dB}$ from 85 to $120 \mathrm{GHz}$ with a chip size of $1.6 \times 1.1 \mathrm{~mm}^{2}$, the signal-signal noise figure is less than $5 \mathrm{~dB}$ from 88 to $95 \mathrm{GHz}$, the saturation output power is $5.8 \mathrm{dBm}$ at $110 \mathrm{GHz}$ and the DC power consumption is $45 \mathrm{~mW}$. The performance of high-gain, wide-band, low noise and medium output power indicates that this MMIC amplifier has a great potential for pre-amplifier or interstage driving amplifiers applications at W-band or D-band. 


\section{Device technology}

More details about the InP PHEMT epitaxial structures, electron mobility and sheet charge concentration were shown in our previously work [12].

The frontside of the InP PHEMT MMIC process provides 200-nm silicon nitride passivated 70-nm T-gate PHEMT devices, $50 \Omega$ /sq thin film resistors (TFR), and $240 \mathrm{pF} / \mathrm{mm}^{2}$ metal-insulator-metal (MIM) capacitors.

In order to reduce the loss at high frequency, the backside of the InP PHEMT MMIC process provides a thinned $50-\mu \mathrm{m}$ thick wafer through substrate vias which connect the backside metal ground plane to the frontside device and circuit elements. In order to minimize source inductance and maintain high device gain, compact $15-\mu \mathrm{m}$ diameter through substrate vias are used with compact $50 \mu \mathrm{m}$ frontside metal pads.

The 70-nm gate InP PHEMT demonstrates an extrapolated cutoff frequency $f_{\mathrm{t}}$ and a maximum oscillation frequency $f_{\max }$ above $200 \mathrm{GHz}$ and $350 \mathrm{GHz}$, respectively. More details about the RF performance of this InP PHEMT were described in reference [12].

\section{The small-signal model and circuit design}

In order to establish the small-signal model accurately from 0.5 to $110 \mathrm{GHz}$, it is essential to obtain the accurate S-parameters of this device. So the test structures, which were introduced just to satisfy the requirements of on-wafer measurement, must be eliminated precisely. In order to improve the de-embedding precision, the open-short method was used to de-embed from 0.5 to $40 \mathrm{GHz}$, and the TRL (ThruReflect-Line) method was adopted from 40 to $110 \mathrm{GHz}$. The measured S-parameters after de-embedding were compared with the model in Fig. 1.

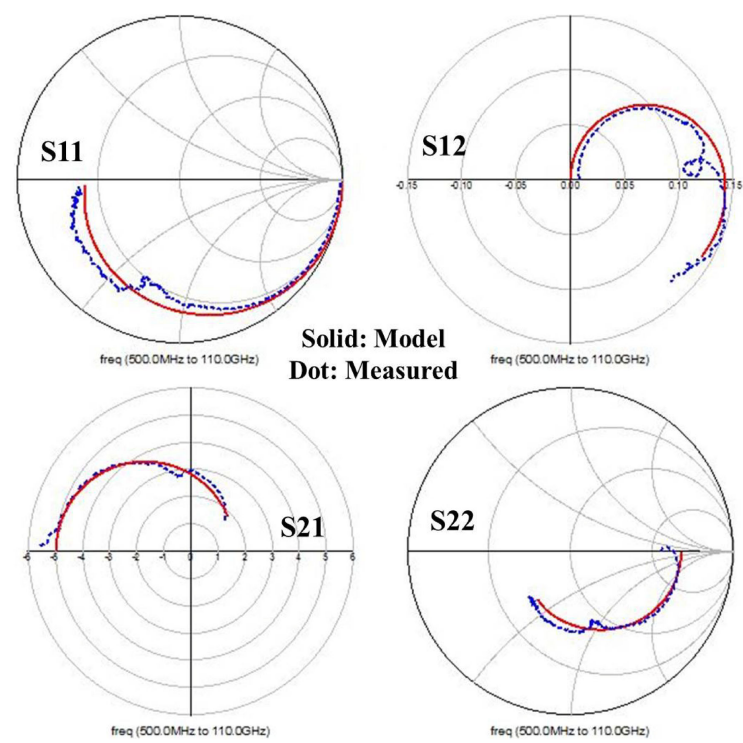

Fig. 1. The measured S-parameters after de-embedding comparison with the model 


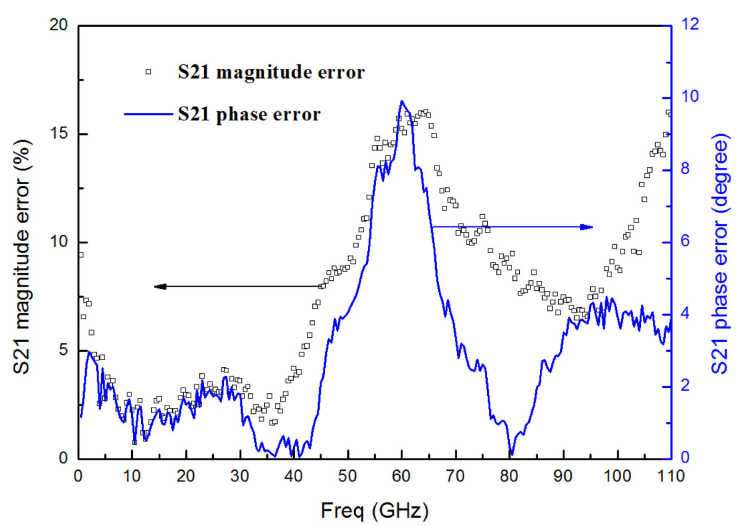

Fig. 2. The S21 errors of the model compared with the measured

A schematic of the design is shown in Fig. 3. Two stages were employed with dual source vias on each device in this MMIC, and every stage used a two-finger PHEMT device, which has $25-\mu \mathrm{m}$ gate width for each finger. RF matching was accomplished adopting high-low impedance microstrip lines on a thinned $50 \mu \mathrm{m}$ thick InP substrate.

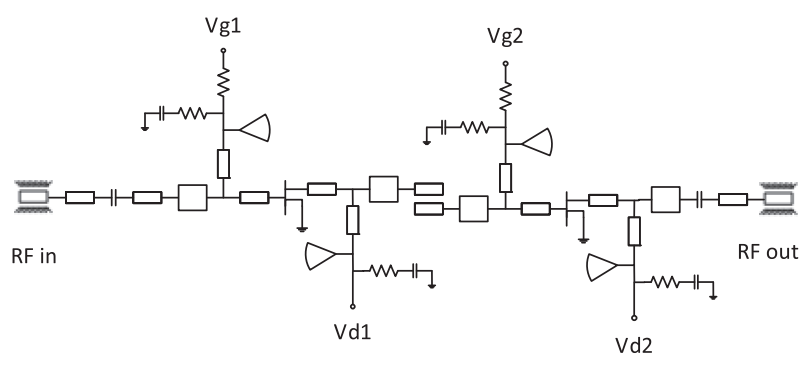

Fig. 3. Schematic of the $85-120 \mathrm{GHz}$ InP MMIC

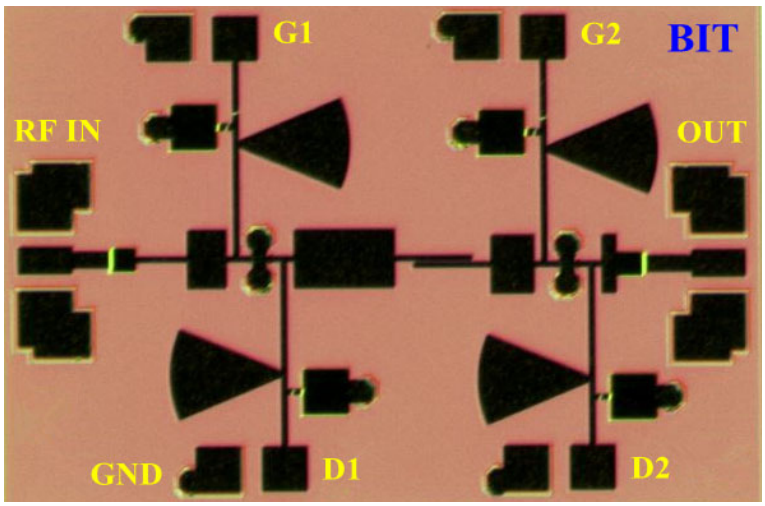

Fig. 4. Chip photograph of the MMIC amplifier

Each stage was designed to have a separate gate and drain bias pad, and quarter-wave shunt stubs were employed as RF shorts to provide bias to the transistors. Edge-coupled lines are used for DC blocking between the first stage and the second stage, radial subs are employed for RF bypass. Shunt RC networks and radial stubs are included in the bias circuitry to maintain amplifier stability. Additionally, the ground coplanar waveguide (GCPW) structure is used in the input 
and output port to make the test easy and convenient. All the passive elements were separately modeled using the ADS Momentum electromagnetic (EM) simulation tool. The photograph of the MMIC is shown in Fig. 4.

\section{On-wafer measurement}

In order to on-wafer measure the small-signal RF performance of this MMIC, we used an Agilent N5245A network analyzer with two frequency extenders from Farran Technology of series FEV-10 (75-110 GHz) and FEV-06 (110-170 GHz) interfaced to WR10 and WR6 waveguides $100-\mu \mathrm{m}$ pitch wafer-probes from Cascade Microtech, respectively. The Line-Reflect-Reflect-Match calibration was used to have the test reference plane at the tip of the probe.

In order to suppress the potential self-oscillations at low frequency which are commonly observed in very high frequency transistors, off-chip bypass capacitors were wire-bonded to every gate and drain bias pad. The simulated S21, the onwafer measured S-parameters and the noise figure (NF) of the MMIC were shown in Fig. 5. The MMIC demonstrated a peak S21 gain of $14.4 \mathrm{~dB}$ at $93 \mathrm{GHz}$ and above $11.5 \mathrm{~dB}$ from 85 to $120 \mathrm{GHz}$ with input return loss of $3-8 \mathrm{~dB}$ and output return loss $7-11 \mathrm{~dB}$ over this band. The $3 \mathrm{~dB}$ bandwidth is above $35 \mathrm{GHz}$.

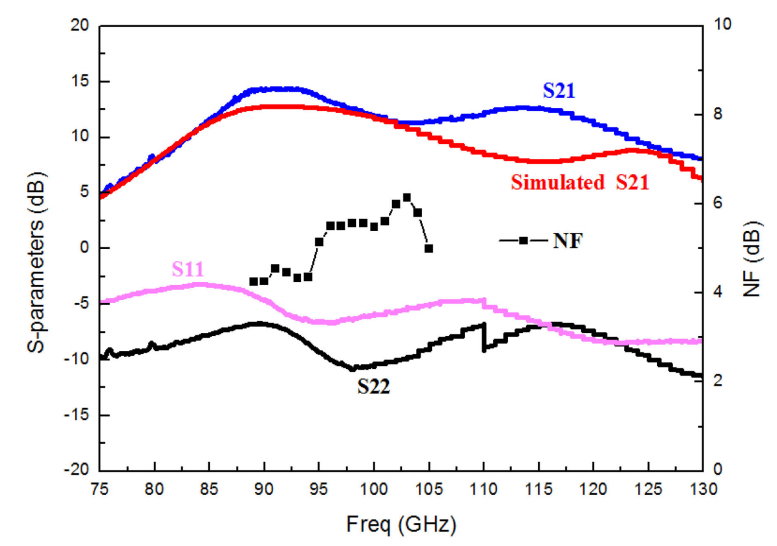

Fig. 5. On-wafer measured S-parameters, simulated S21 and the NF curves

As shown in Fig. 5, the measured S21 curve is same as the simulated from 75 to $85 \mathrm{GHz}$ and its value is slightly higher than the simulated from 85 to $105 \mathrm{GHz}$ with the same trend, however, from 105 to $125 \mathrm{GHz}$, a bigger disagreement occurs. The discrepancy between the simulated and the measured S21 can be attributed to two factors. The first one is the model errors as shown in Fig. 2, the S21 magnitude relative error is less than $5 \%$ from 3 to $42 \mathrm{GHz}$ and less than $10 \%$ in the band of $0 \sim 3 \mathrm{GHz}, 43 \sim 52 \mathrm{GHz}$ and $76 \sim 102 \mathrm{GHz}$. However, it is above $10 \%$ and less than $16 \%$ in the band $52 \sim 76 \mathrm{GHz}$ and $102 \sim 110 \mathrm{GHz}$, the $\mathrm{S} 21$ phase error is less than $5^{\circ}$ except for the band 52 66 GHz. Big errors at $102 \sim 110 \mathrm{GHz}$ can be explained as follows, the test curve at high frequency is not smooth as it at low frequency, it would be smoothed for modeling, and the model precision is decreased with the bandwidth increasing, we should make a tradeoff between the model precision and bandwidth. The second factor is that the model is based on the measured S- 
parameters from 0.5 to $110 \mathrm{GHz}$, however the model performance beyond $110 \mathrm{GHz}$ is extrapolated.

Due to the test equipment restrictions, the NF was measured only from 88 to $105 \mathrm{GHz}$, as shown in Fig. 5, it was less than $5 \mathrm{~dB}$ from 88 to $95 \mathrm{GHz}$, and less than $6 \mathrm{~dB}$ from 95 to $105 \mathrm{GHz}$. Meanwhile, the saturation output power was $5.8 \mathrm{dBm}$ at $110 \mathrm{GHz}$ with input power of $0 \mathrm{dBm}$ and the DC power consumption is $45 \mathrm{~mW}$ $(1.5 \mathrm{~V} \times 30 \mathrm{~mA})$.

The comparisons of this work with the commercial products and previously published results were shown in Table I. Our MMIC amplifier has much highergain per stage and wider-band characteristics than others at the similar frequency band. The performance of high-gain, wide-band, low power consumption and medium output power indicates that this MMIC amplifier has a great potential for pre-amplifier or interstage driving amplifiers applications at W-band or D-band.

Table I. The performance comparisons of this work with others

\begin{tabular}{ccccccc}
\hline Comp & $\begin{array}{c}\text { Freq } \\
(\mathrm{GHz})\end{array}$ & $\begin{array}{c}\text { Gain/stage } \\
(\mathrm{dB})\end{array}$ & $\begin{array}{c}3 \mathrm{~dB} \\
\text { bandwidth } \\
(\mathrm{GHz})\end{array}$ & $\begin{array}{c}\mathrm{NF} \\
(\mathrm{dB})\end{array}$ & $\begin{array}{c}\text { P-1 dB } \\
(\mathrm{dBm})\end{array}$ & $\begin{array}{c}\text { DC } \\
\text { power } \\
(\mathrm{mW})\end{array}$ \\
\hline HRL [4] & $75-110$ & 5.5 & 35 & 3.5 & 0 & 42 \\
\hline HRL [5] & $75-103$ & 5.7 & 28 & 8 & $6 \sim 7$ & 240 \\
\hline TRW [6] & $110-130$ & 5 & 20 & $3.8 \sim 4.5$ & - & - \\
\cline { 2 - 8 } & $113-119$ & 5.7 & 6 & $3.8 \sim 4.5$ & - & - \\
\hline TRW [7] & $110-120$ & 6 & 10 & 6.3 & - & 90 \\
\hline OMMIC [8] & $80-100$ & 6.7 & $<20$ & $2 \sim 4$ & - & - \\
\hline TRW [9] & $85-115$ & 4.3 & 30 & $3 \sim 4$ & - & - \\
\hline CETC [10] & $80-102$ & 3.8 & 22 & 5 & - & 30 \\
\hline OMMIC [13] & $75-110$ & 5.8 & 35 & 3 & 1 & 33 \\
\hline This work & $\mathbf{8 5 - 1 2 0}$ & $\mathbf{7}$ & $\mathbf{3 5}$ & $\mathbf{4 \sim 6}$ & $\mathbf{4}$ & $\mathbf{4 5}$ \\
\hline
\end{tabular}

\section{Conclusion}

The design, fabrication and on-wafer measurement of a two-stage $85-120 \mathrm{GHz}$ wide-band MMIC amplifier have been described, which demonstrated a smallsignal peak gain of $14.4 \mathrm{~dB}$ at $92 \mathrm{GHz}$ and greater than $11.5 \mathrm{~dB}$ from 85 to $120 \mathrm{GHz}$ with $3 \mathrm{~dB}$ bandwidth of above $35 \mathrm{GHz}$ and a chip size of $1.6 \times 1.1 \mathrm{~mm}^{2}$. The NF was less than $5 \mathrm{~dB}$ from 88 to $95 \mathrm{GHz}$, meanwhile, the saturation output power was $5.8 \mathrm{dBm}$ at $110 \mathrm{GHz}$ and the DC power consumption is $45 \mathrm{~mW}$. So this MMIC amplifier has a great potential for W-band or D-band applications.

\section{Acknowledgement}

This research is supported by the National Natural Science Foundation of China (No. 61275107). The authors would like to thank the members of Hebei Semiconductor Research Institute for their help on MMIC fabrications and the 75-110 GHz, 110-170 GHz on-wafer measurement. 